\title{
perifèria
}

Número 18(2), diciembre 2013

http://revistes.uab.cat/periferia

\section{L'agro-pastoralisme mobile des agdals du Haut Atlas}

\author{
Pablo Dominguez - University of Kent - Centre for Biocultural Diversity ${ }^{1}$
}

\section{Resumen}

Cette étude décrit I'agro-pastoralisme mobile des Aït Ikiss, communauté du Haut Atlas de Marrakech. La transhumance sur laquelle se base cet agro-pastoralisme mobile des Aït Ikiss est particulièrement imbriquée au sein d'un système d'agdals. Ce système d'agdals consiste à interdire le pâturage en différents habitats et à différentes époques de l'année, pour créer des espaces pastoraux complémentaires (notamment abondants en ressources herbacées), en permettant un repos aux différentes strates de végétation, ainsi que l'établissement de jeunes semis, et donc le maintien de l'activité agro-pastorale et des écosystèmes qui soutiennent cette communauté des Ait Ikiss à travers les générations.

\begin{abstract}
This study describes the mobile agro-pastoralism of the Ait Ikiss, community of the High Atlas of Marrakech. The transhumance on which is based the mobile agropastoralism of the Ait Ikiss is particularly nested within a system of agdals. This agdals system consists on prohibiting grazing in different habitats and at different times of the year to create additional pastoral areas (particularly abundant in herbaceous resources), allowing a rest to different strata of vegetation, as well as the establishment of seedlings, thus maintaining the agro-pastoral activity and ecosystems that sustain this community of the Ait Ikiss through the generations.
\end{abstract}

\section{Introduction}

Chez les Aït Ikiss, communauté d'environ 700 personnes appartenant à la tribu des Mesioua et occupant un peu plus d'une vingtaine de Kilomètres carrés dans le Haut Atlas de Marrakech, entre 1.200 et 3.000 mètres d'altitude, la transhumance peut être définie comme un véritable système de gestion communautaire des ressources sylvo-pastorales. Comme dans d'autres communautés du Haut Atlas (Mahdi 1999), elle est intimement liée à la prohibition agro-pastorale de l'agdal, pratique qui dans cette région consiste en une mise en défens d'un territoire ou ressource donnés

\footnotetext{
${ }^{1}$ Enviar correspondencia a: Pablo Dominguez eco.anthropologies@gmail.com
} 


\section{perifèria}

Número 18(2), diciembre 2013

http://revistes.uab.cat/periferia

durant une période concrète permettant la reconstitution des réserves végétales, la floraison et l'établissement des semis et en fin, la continuité de l'écosystème et de l'activité pastorale. Cette prohibition est décidée par la jmaa, l'assemblée coutumière composée par les chefs de famille, afin de permettre la régénération et l'exploitation durable des ressources naturelles tout en assurant un accès relativement égalitaire à tous les ayants droit. Les Aït Ikiss associent les déplacements de leurs animaux, ainsi que de tout le groupe, au calendrier des mises en défens des différents habitats dont ils sont les détenteurs. De fait, dans leur système de transhumance, ils combinent jusqu'à 5 agdals interdisant le pâturage de différents espaces à différents moments de l'année en combinaison avec d'autres agdals concernant les productions agricoles et fruitières comme I'on verra plus tard.

Le pastoralisme mobile de la transhumance est une pratique très répandue dans I'ensemble du Maghreb, et sa mise en œuvre au sein de l'organisation coutumière est intéressante à plusieurs titres. Sur le plan du fonctionnement agro-écologique, elle permet d'étager les pâturages au cours des saisons, laissant certains terroirs se reposer pendant qu'on utilise un ou deux d'entre eux. Cette complémentarité des différents cycles végétaux est notamment fonction des étages altitudinaux et écologiques (Alaoui 2009), permettant une plus grande productivité des pâturages disponibles à chaque époque (Bourbouze 1999).

Sur le plan économique, la transhumance a une grande importance pour les populations d'agropasteurs, notamment dans la haute montagne, où les déplacements sont nécessaires si on veut mettre en valeur toute la potentialité agro-pastorale. Par exemple, chez les Mesioui, sans ce système agropastoral fondé sur l'agdal et les déplacements du grand nombre de troupeaux rattachés à presqu'une centaine de villages, l'équilibre socio-écologique et économique de ces populations s'écroulerait (Dominguez 2010). La transhumance, avec ses pactes d'usage et de transit, telle qu'elle est pratiquée dans la haute vallée du Zat, dont les Aït Ikiss font partie, est à la fois une manière de maintenir la ressource et une façon d'assurer la paix entre les bergers. 


\section{revista de recerca i formació en antropologia}

\section{perifèria}

Número 18(2), diciembre 2013

http://revistes.uab.cat/periferia

$\mathrm{Au}$ niveau socioculturel, l'ouverture de différents agdals pastoraux et la conséquente transhumance qui s'y démarre juste après, est l'occasion d'un intense déploiement d'activité rituelle et symbolique, allant de pair avec le calendrier agropastoral (Mahdi \& Dominguez 2009). Les aspects symboliques ne peuvent pas être ici négligés comme si la transhumance pouvait continuer d'exister telle qu'elle est, indépendamment de tout son héritage culturel, au sein duquel elle est née et elle s'est construite jour à jour. La transhumance met en branle la presque totalité de la communauté et sert d'écran où se projette une grande partie de ses valeurs et de son système culturel.

En fin, la transhumance chez les Aït Ikiss, comme dans nombreuses autres communautés Atlasiques, implique donc à la fois des notions géographiques et territoriales, des connaissances profondes sur les ressources naturelles disponibles, des modes de gestion de la concurrence sociale, et des systèmes de représentation qui prennent leurs racines dans une culture berbère ou amazigh millénaire. 


\section{perifèria}

Número 18(2), diciembre 2013

http://revistes.uab.cat/periferia

\section{Contexte de l'étude: Organisation et utilisation des espaces agro-pastoraux}

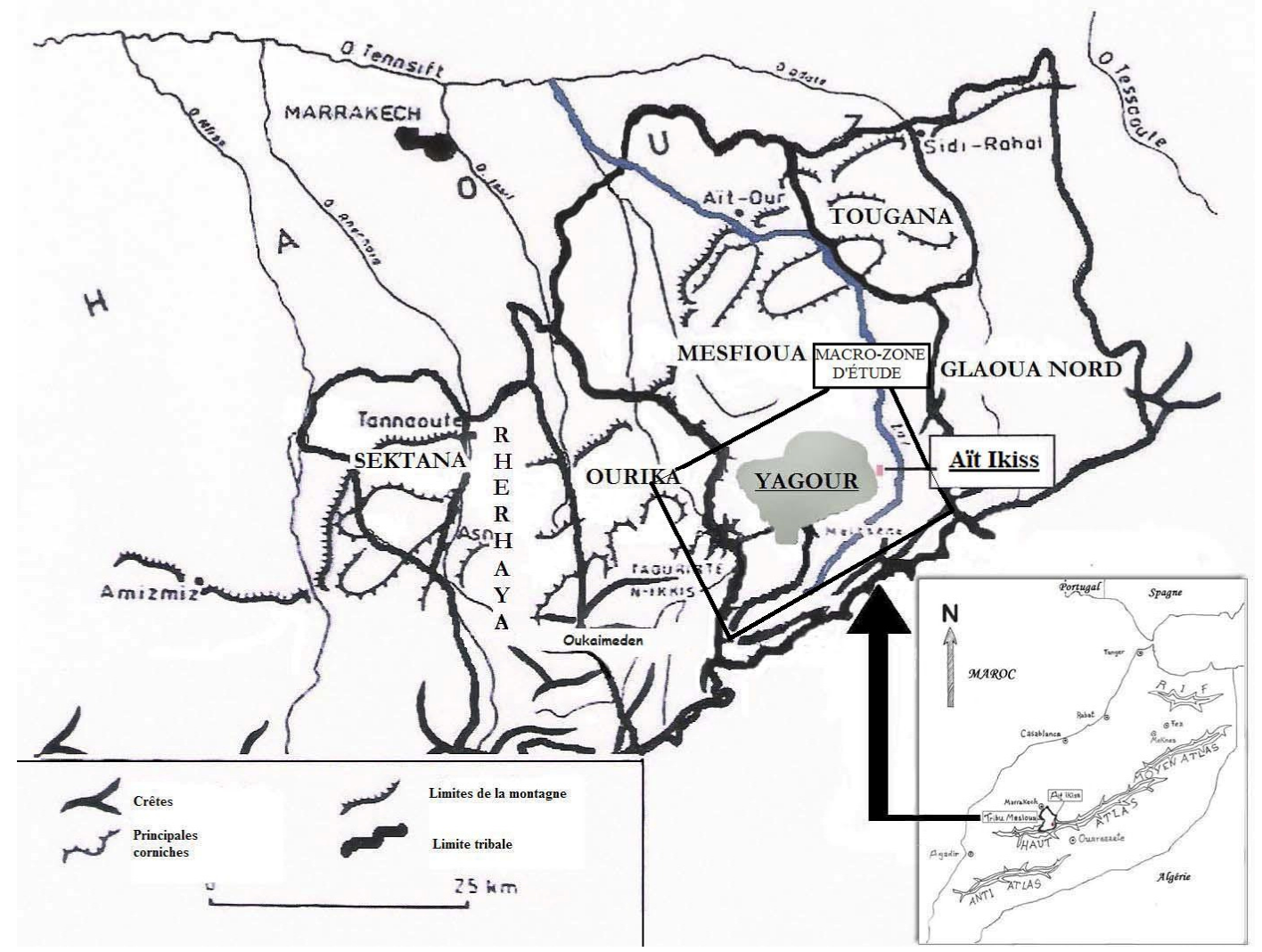

Figure 1

Situation du territoire des Aït Ikiss au sein de la tribu Mesioua et à côté du grand territoire pastoral du Yagour (à partir de LAFUENTE F., 1968).

Cette étude a été conduite dans la région la plus montagneuse de la tribu Mesioua dont font partie les Aït Ikiss, communauté que nous avons étudiée plus en profondeur. Elle est située à 50 kilomètres de Marrakech à vol d'oiseau (Maroc central) et celle-ci est incluse dans la commune rurale de I'Arbaa Tighdouine. Au sein de cette région se trouve le Yagour, cœur névralgique du territoire des Mesioui de la montagne. Il s'agit d'un territoire pastoral situé entre 1.900 et 3.600 mètres d'altitude, ayant une superficie d'environ $70 \mathrm{Km}^{2}$ et autour duquel se déplacent les 5 fractions tribales existant dans notre macro-zone d'étude. Près de 7.500 


\section{perifèria}

Número 18(2), diciembre 2013

http://revistes.uab.cat/periferia

personnes appartenant à une cinquantaine de villages et hameaux dépendent de façon directe de la transhumance au Yagour. L'accès aux pâturages du Yagour, élément d'un système agro-sylvo-pastoral plus large et plus complexe, est interdit par l'institution de l'agdal, du 28 mars jusqu'au début de l'été. La date exacte d'ouverture de l'agdal du Yagour dépende de la pluviométrie annuelle (a grosso modo le plus humide l'année le plus tardive l'ouverture). Le Yagour a une importance particulière qui découle de sa grande abondance d'herbe et sa grande taille (dans la langue local tachelhit, Yagour veut dire «plus grand que ») et qui conditionne les dates d'ouverture estivale de beaucoup d'autres grands agdals voisins (Tiferwan, Tisiyi, haut Zat, etc.).

Les Mesioui gardent plus ou moins les marques de l'ancienne organisation tribale, qualifiée de segmentaire par l'anthropologie britannique (Gellner 1969), étant caractérisée par un emboîtement de différents groupes sociaux (les « segments »), agencés à la manière d'un jeu de poupées russes. Par exemple, la partie la plus montagnarde de la tribu Mesioua, se divise en fractions, étant la fraction Aït Zat celle à laquelle appartiennent les Aït Ikiss, qui a leur tour continuent de s'organiser encore en sous-villages, lignages et familles nucléaires. Dans ce contexte, les jemaas, des assemblées coutumières, organisent leurs activités collectives: transhumance, travaux collectifs, règlements d'irrigation, droits de pâturage, application des différents agdal, organisation des fêtes, célébrations religieuses, etc. 


\section{perifèria}

Número 18(2), diciembre 2013

http://revistes.uab.cat/periferia

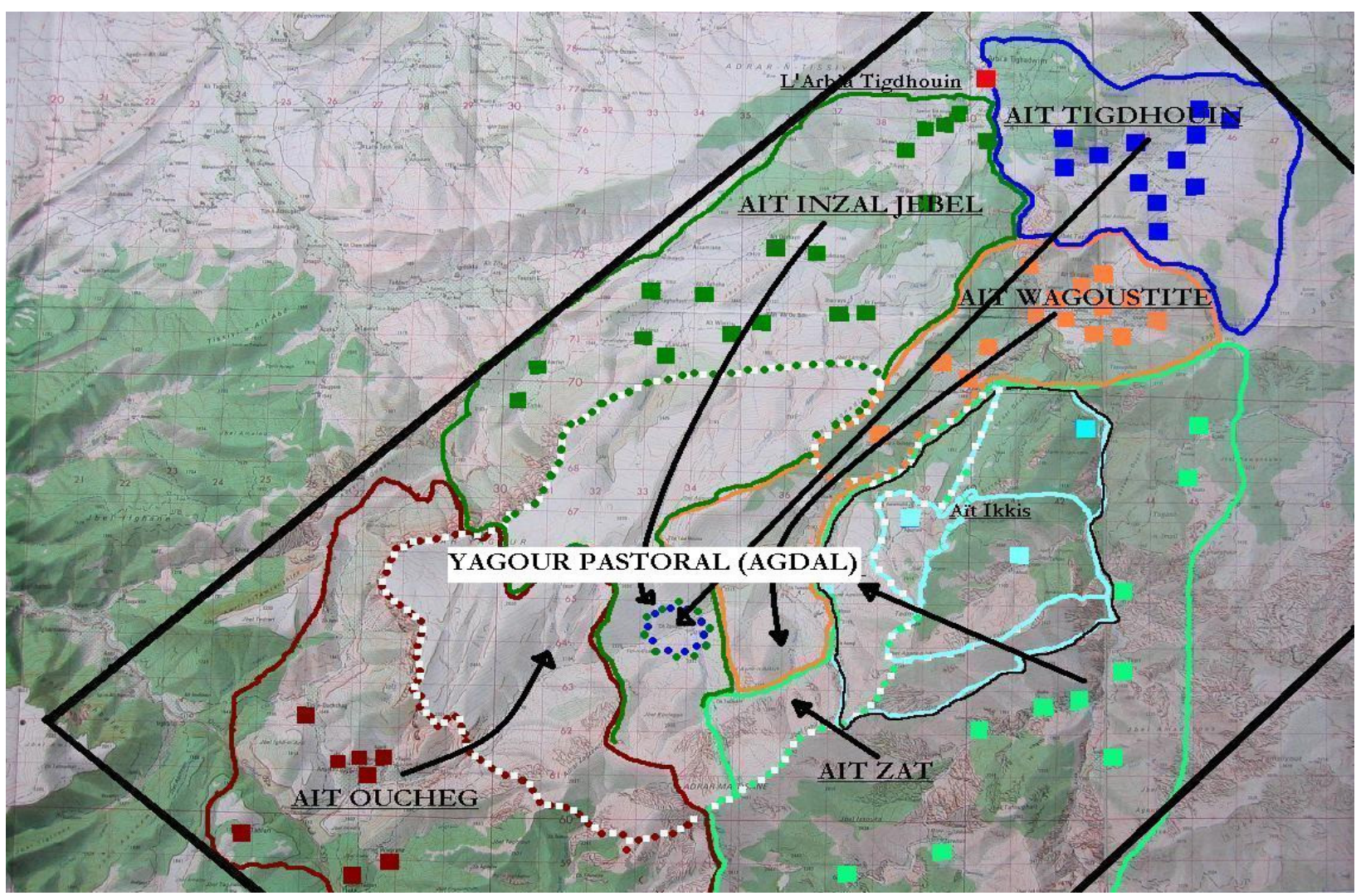

Figure 2

Carte des fractions et douars des hauts Mesioui, et voies principales de transhumance

3. Importance et déroulement de la transhumance chez les Aït Ikiss : le labyrinthe organisé des tagdalts.

La communauté Aït Ikiss occupe quatre habitats différents selon la séquence altitudinale suivante: Azgour/Tifni, Ikiss, Warzarzt et Yagour d'Ikiss. Proportionnellement à leur nombre, les Aït Ikiss utilisent une plus grande partie du Yagour que les autres communautés des Mesioui. Pour expliquer cette situation, ils invoquent un épisode héroïque (historique ou légendaire) qui leur aurait donné droit à une plus grande superficie de terre sur le Yagour. 


\section{perifèria}

Número 18(2), diciembre 2013

http://revistes.uab.cat/periferia

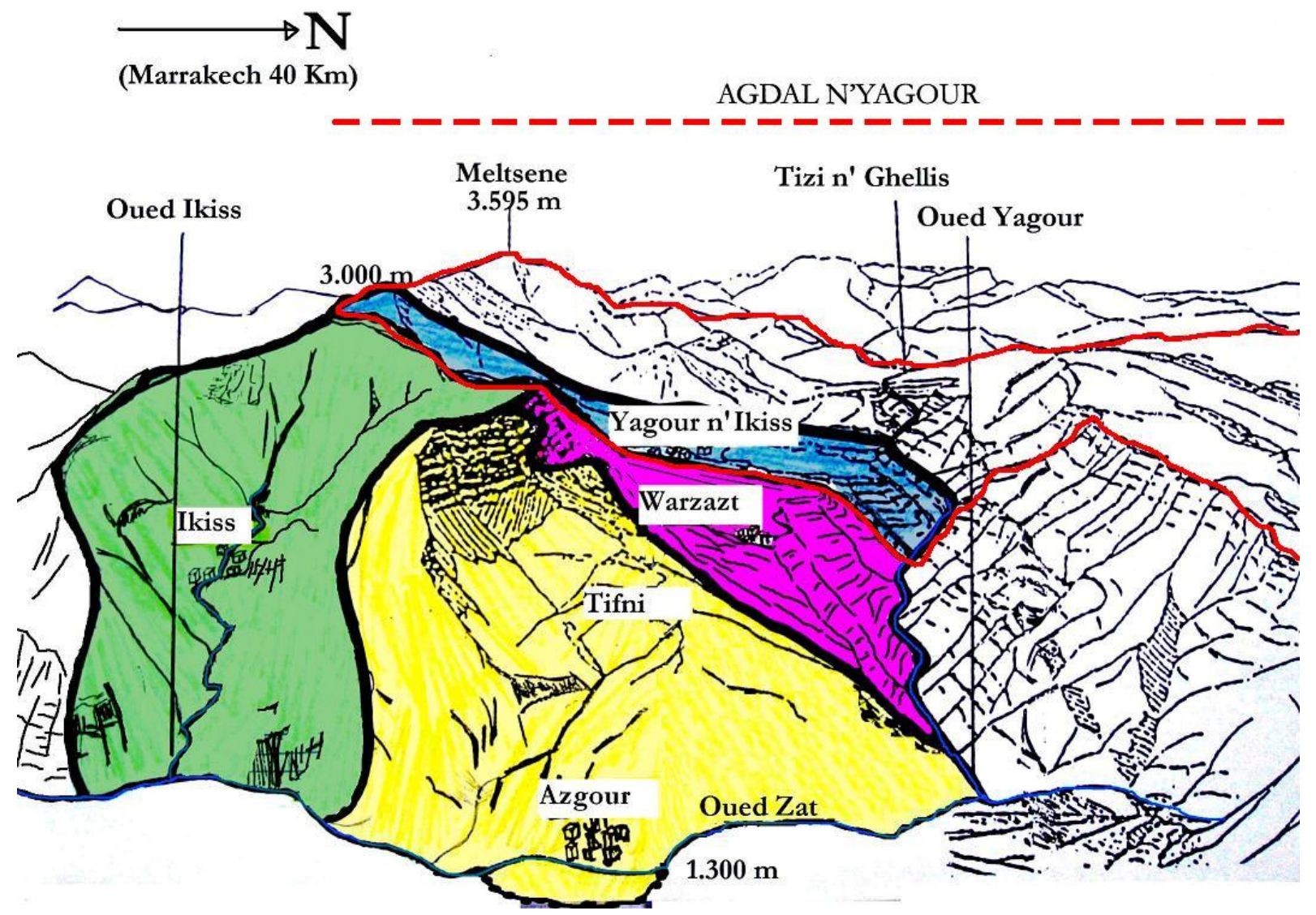

Figure 3

Territoire des Aït Ikiss et ses quatre habitats

Sur le territoire des Aït Ikiss, la topographie est très diverse (grands escarpements, nombreux plateaux, étroits fonds de vallée, etc.) et les variations du climat se traduisent pour les troupeaux par des périodes de disette, notamment en été, du fait de la sécheresse, et en hiver du fait du froid rigoureux. Il s'agit donc pour ces agropasteurs de raisonner l'utilisation de leur territoire dans le temps et dans l'espace, afin de répondre aux besoins alimentaires des troupeaux et d'assurer des transferts de fertilité vers les zones cultivées (Genin et al 2013). Pour ce faire, ils ont mis en place un système complexe et ingénieux de transhumances, réglementé 


\section{perifèria}

Número 18(2), diciembre 2013

http://revistes.uab.cat/periferia

par l'imposition des différents agdals. Dans cette partie, nous tenterons de mettre en lumière toute l'importance du pastoralisme mobile des agdals pour la survie du groupe. Ainsi, nous expliquerons les différents choix de mises en défens (agdals) rythmant la transhumance des troupeaux et les déplacements des familles, tout en nous intéressant à leur complémentarité avec l'évolution des ressources fourragères, agricoles et fruitières au cours de l'année. Une série des schémas expliquent le système d'ouverture et de fermeture des différents agdals dans leurs 4 habitats, sur lequel se calque la transhumance Aït Ikiss.

au 28 Mars

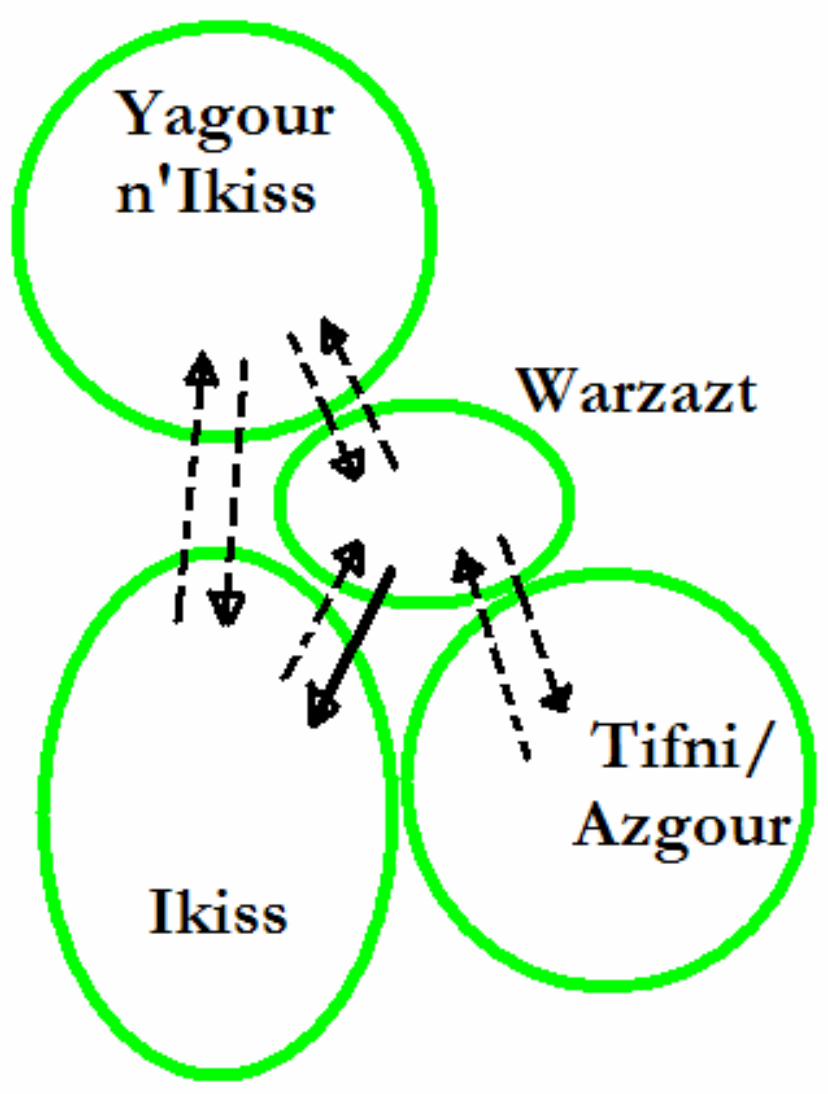

Tous les espaces sont ouverts en début d'automne et les mouvements sont multiples. Mais fondamentalement c'est la cueillette des noix en fin septembre qui commande les mouvements (le déplacement principal est toujours signalé avec une flèche foncée). La plupart des gens partent à Ikiss car c'est là que se trouvent la plus grande partie des noyers. Le 28 septembre s'ouvre l'agdal d'Ikiss qui interdisait la cueillette des noix ainsi que de quelques autres fruits. Ikiss est I'habitat le moins haut donc le moins froid. C'est le village d'origine de tout le groupe qui y dispose des habitations les plus nombreuses et les mieux 


\section{revista de recerca i formació en antropologia}

\section{perifèria}

Número 18(2), diciembre 2013

http://revistes.uab.cat/periferia

isolées pour passer I'hiver. Au moment de leur retour, les habitants d'Ikiss célèbrent un maarouf, un sacrifice de bêtes et repas collectif en I'honneur de la sainte locale d'Afoud ${ }^{2}$, pour s'assurer un bon hiver.

au 20 Avril environ

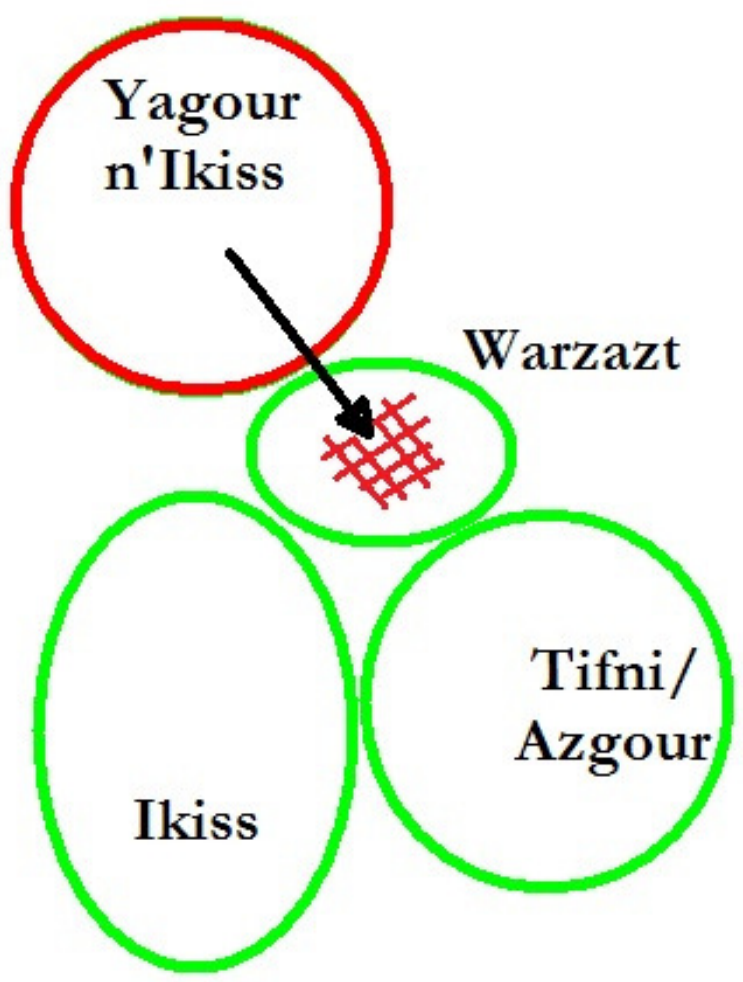

L'ensemble du Yagour est mis en agdal pour favoriser la croissance de la végétation, notamment les herbacées qui à la fin de la prohibition d'accès de l'agdal, atteignent plus d'un mètre de hauteur en bonne année sur les sols les plus favorables. Cent ou deux cents bergers de toute la tribu Mesioua, dont une partie appartenant aux Aït Ikiss, doivent en sortir du territoire et retourner à leur village d'origine ou sur des pacages de printemps situés plus bas. Le Yagour est ainsi vidé de tous ses utilisateurs durant approximativement trois

mois. Au même moment, un autre petit agdal est décrété, cette fois sur les bandes herbacées divisant les différentes parcelles agricoles, au sein de l'espace « ouvert » de Warzazt.

${ }^{2}$ Curieusement, et malgré avoir demandé nombreuses fois, personne n'a pas su donner le nom de la sainte ni son hagiographie, et elle est simplement connue et nommée comme la sainte d'Afoud, un des 5 sous-douars du village d'Ikiss. 


\section{perifèria}

Número 18(2), diciembre 2013

http://revistes.uab.cat/periferia

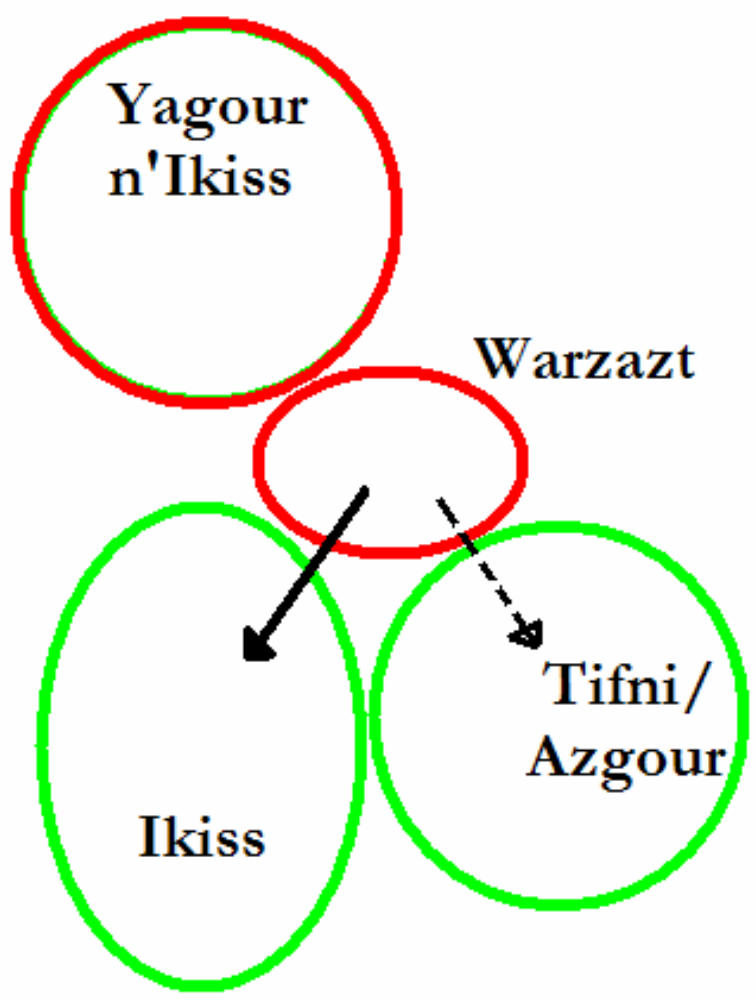

Au début du printemps, Warzarzt, deuxième habitat le plus haut des Aït Ikiss, ferme aussi tout son espace pour laisser repousser I'herbe sur les versants et les petits plateaux. Le mois de mai est en effet, selon les éleveurs et bergers, le moment le plus sensible de pousse des herbacées à Warzazt. La vingtaine de familles qui ont leur maison principale à Warzarzt (surtout des familles d'éleveurs d'ovins), se voient cette fois-ci obligées de transhumer avec leur bétail vers les bergeries de Tifni (sur le chemin au hameau d'Azgour), et vers la vallée d'Ikiss où se trouvent les bergeries de printemps les plus basses.

\footnotetext{
${ }^{3}$ Ces dates varient chaque année en fonction des différentes négociations.
} 


\section{perifèria}

Número 18(2), diciembre 2013

http://revistes.uab.cat/periferia

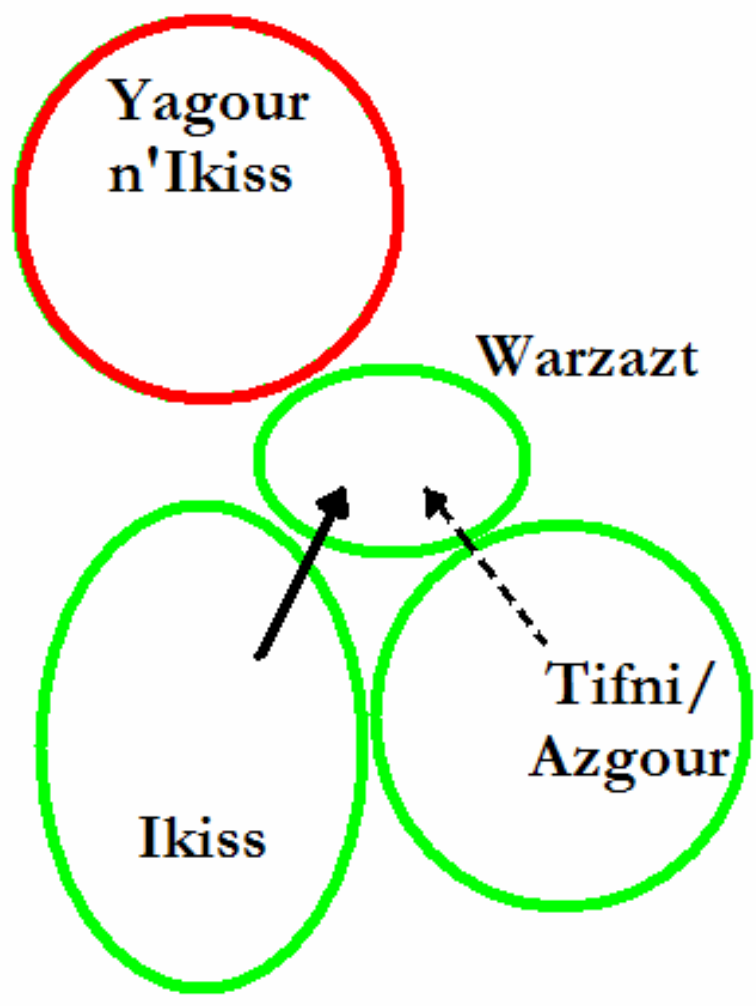

Au bout d'un mois (autour du 20 mai), l'espace de Warzarzt est ouvert. Cela produit une migration inverse : les gens montent d'Ikiss et de Tifni vers Warzarzt, où commence à peu près un mois plus tard (vers la fin juin), la récolte de I'orge. Alors la presque totalité des Aït Ikiss part en transhumance vers les hauteurs. Les deux boutiques de Warzarzt qui étaient fermées depuis septembre ouvrent et le fqih se déplace aussi avec le groupe jusqu'à Warzazt. Ce déplacement vers le haut symbolise le début de l'été pour les Aït Ikiss. Aussi, juste avant de monter, ils célèbrent un maarouf à Ikiss, dans l'espoir d'obtenir la bénédiction d'Allah pour un bon séjour sur les estives par l'intermédiaire de la sainte dont le tombeau se trouve à Ikiss. 


\section{perifèria}

Número 18(2), diciembre 2013

http://revistes.uab.cat/periferia

au 28 Septembre

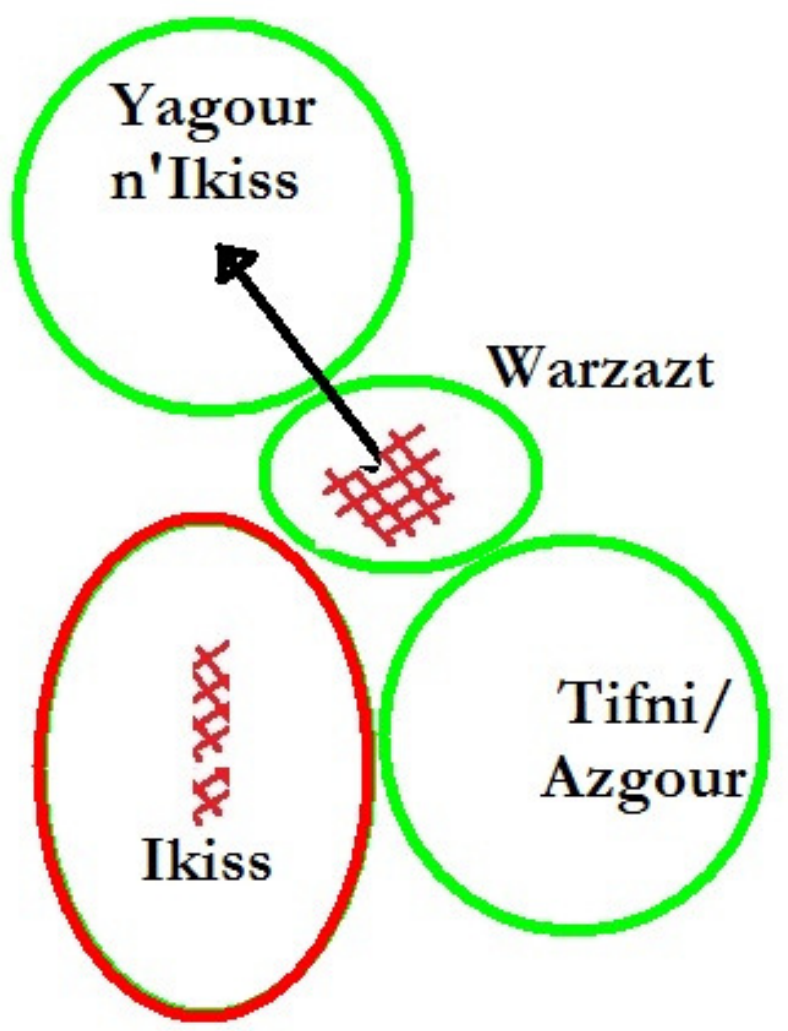

Après environ trois mois de mise en défens de tous les territoires d'altitude des Mesioui de la montagne, le Yagour ouvre. En fonction des conditions écologiques annuelles (notamment la pluviométrie), cette ouverture se fait plus ou moins tôt, théoriquement selon la décision prise par la jmaa de l'ensemble des Mesioui. Les bergers transhumants, Aït Ikiss et des autres fractions, provenant de tous les territoires contigus au Yagour (tant des vallées de I'Ourika que du Zat), remontent les versants conduisant aux riches pâturages d'été. L'ouverture de l'agdal du

Yagour est un événement très important localement parce que c'est un grand lieu de rassemblement des troupeaux, mais aussi des gens pendant une grande partie de l'année... Des maarouf et d'autres événements festifs ou rituels ont lieu à ce moment-là. Dans les zones favorables à l'agriculture, mises en culture, la récolte des céréales a lieu quelques jours avant l'ouverture du Yagour ou dans les semaines qui suivent. Par ailleurs, vers le début de juillet, le fond de la vallée d'Ikiss est mis en agdal et personne ne peut plus y faire pâturer de bêtes, notamment dans le but de protéger les arbres fruitiers, dont leurs fruits sont euxmêmes interdits d'être cueillis jusqu'à l'ouverture de l'agdal d'Ikiss. Vers la mijuillet est aussi imposé un autre agdal sur l'ensemble de la vallée d'Ikiss, au-delà du fond de la vallée, dans le but de diminuer la pression sur les pâturages des versants durant la période critique de l'été et ceci jusqu'au retour à Ikiss pour la 


\section{perifèria}

Número 18(2), diciembre 2013

http://revistes.uab.cat/periferia

cueillette des noix en fin Septembre, rénovant ainsi le cycle commencé un an avant.

\section{Bibliografía}

Alaoui, Safia (2009). Les pelouses humides dans le haut Atlas : Biodiversité végétale, dynamique spatiale et pratiques de gestion coutumièr. Marrakech: Thèse de Doctorat de I'Université Cadi Ayyad.

Auclair, Laurent \& AlIfriqui, Mohamed (coord.) (2012). Agdals. Société et gestion des ressources dans I'Atlas marocain. Rabat: Ed. Institut Royal de la Culture Amazighe - Institut de Recherche pour le Développement.

Bourbouze, AlAIN (1999). "Gestion de la mobilité et résistance des organisations pastorales des éleveurs du Haut Atlas marocain face aux transformations du contexte pastoral maghrébin", en Managing mobility in african rangeland : the legitimization of transhumance. Beijer: FAO and Beijer intern. instit. Ecolog. Economics, pp. 236-265.

Dominguez, Pablo (2010). Approche multidisciplinaire d'un système traditionnel de gestion des ressources naturelles communautaires: L'agdal pastoral du Yagour (Haut Atlas marocain). Barcelona: Thèse de Doctorat, École des Hautes Études en Sciences Sociales, Paris, France / Universitat Autònoma de Barcelona.

Gellner, Ernest (1969). Saints of the Atlas. London: Ed. Weidenfield and Nicholson.

Genin Didier, Fouilleron Benjamin y Kerautret Loic (2012). "Un tempo bien tempéré... Place et rôles des agdals dans les systèmes d'élevage des Aït Bouguemez (Haut Atlas central)" en Agdals. Société et gestion des ressources dans l'Atlas marocain. Rabat: Ed. Institut Royal de la Culture Amazighe - Institut de Recherche pour le Développement.

Mahdi, Mohamed (1999). Pasteur de l'Atlas. Production pastorale, droit et rituel. Casablanca: Ed. Fondation Adenauer.

Mahdi, Mohamed y Dominguez, Pablo (2009). "Regard anthropologique sur transhumance et modernité au Maroc", AGER, no 8, pp. 45-73. 